\title{
Comparison of Perioperative Outcomes Between Laparoscopic and Open Partial Nephrectomy for Different Complexity Renal Cell Carcinoma Based on the R.E.N.A.L. Nephrometry Score
}

\author{
Yang $\mathrm{Yu}^{\mathrm{l}, *}$ \\ Wenfeng Wang ${ }^{2} *$ \\ Zuquan Xiong ${ }^{3}$ \\ Zhenyu Yang' \\ Jun $\mathrm{Li}^{\mathrm{I}}$ \\ Yifan Shen' \\ Bin $\mathrm{Gu}^{\prime}$ \\ 'Urology Department, Shanghai Pudong \\ New Area People's Hospital, Shanghai, \\ People's Republic of China; ${ }^{2}$ Cancer \\ Institute, Fudan University Shanghai \\ Cancer Center, Fudan University, \\ Shanghai, People's Republic of China; \\ ${ }^{3}$ Urology Department, Shanghai Huashan \\ Hospital, Shanghai, People's Republic of \\ China
}

*These authors contributed equally to this work
Correspondence: Bin Gu Urology Department, Shanghai Pudong

New Area People's Hospital, 490 Chuanhuan South Road, Shanghai, 20I200, People's Republic of China $\mathrm{Tel} / \mathrm{Fax}+86-2120509000$

Email ibippan3998@I63.com
Purpose: This study aimed to compare perioperative outcomes of open partial nephrectomy (OPN) and laparoscopic partial nephrectomy (LPN) from a retrospective single-center dataset.

Patients and Methods: A retrospective single-center analysis of 210 patients who underwent open $(n=91)$ or laparoscopic $(n=119)$ partial nephrectomy for RCC was conducted between 2012 and 2015. All patients were grouped into low complexity, moderate complexity, and high complexity according to the R.E.N.A.L. Nephrometry Score, respectively. The rates of intraoperative and postoperative complications estimated blood loss, warm ischemia time, operative time, conversion of laparoscopic procedure to open surgery, and postoperative length of stay were assessed for both procedures.

Results: In low complexity group ( $\mathrm{n}=93)$, patients undergoing LPN ( $\mathrm{n}=52)$ under ischemia conditions had significantly longer renal artery clamp time $(p<0.001)$ and operative time $(p$ $=0.001)$ compared with OPN $(n=41)$. However, patients undergoing LPN had a significantly less postoperative length of stay $(\mathrm{p}=0.005)$ and estimated blood loss $(\mathrm{p}<0.001)$ compared with OPN. There was no statistically significant difference in the rates of complications between LPN and OPN. In the moderate complexity group $(n=114), 67$ and 47 patients underwent LPN and OPN, respectively. LPN had notably longer warm ischemia time ( $\mathrm{p}<$ $0.001)$ and operative time $(\mathrm{p}<0.001)$ compared with OPN. There were no statistically significant differences in the rates of complications, estimated blood loss, and postoperative length of stay between LPN and OPN. In the high complexity group $(n=3)$, all patients underwent OPN.

Conclusion: OPN and LPN procedures performed in patients with low and moderate complexity tumors based on the RENAL Nephrometry score offer acceptable and comparable results. When applied to low complexity tumors, our data suggest that laparoscopic NSS is an effective, minimally invasive therapeutic approach with the advantages of less blood loss, earlier hospital discharge, and more rapid convalescence.

Keywords: renal cell carcinoma, open partial nephrectomy, laparoscopic partial nephrectomy, R.E.N.A.L. Nephrometry Score

\section{Introduction}

Over the past 2-3 decades, the incidence of kidney cancer has steadily increased all around the world. ${ }^{1}$ The EUA Guidelines suggested that surgery is the only curative treatment for localized renal cell carcinoma $(\mathrm{RCC}){ }^{2,3}$ The partial nephrectomy 
(PN) and radical nephrectomy (RN) for patients with ccRCC larger than $7 \mathrm{~cm}$ exhibited equivalent oncologic outcomes. ${ }^{4}$ Basing on quality-of-life and oncological outcomes, localized cT1 carcinomas are best treated through nephron-sparing surgery (NSS) rather than RN. ${ }^{5,6}$ The American Urological Association and European Association of Urology claim NSS as the operation of choice when feasible. ${ }^{7,8}$ The average estimated blood loss of the laparoscopic approach was universally lower open approach, but warm ischemia time (WIT) was longer. ${ }^{9,10}$ In a matched-pair study, the renal function was poorer in the laparoscopic PN cohort in the shortterm after surgery. ${ }^{11}$ There is no difference in PFS or OS between laparoscopic PN (LPN) and open PN (OPN) with laparoscopic expertise. ${ }^{12,13}$ NSS can be executed, either with an open or laparoscopic approach, basing on the surgeon's expertise and skills and utilizable equipment. ${ }^{14,15}$

The R.E.N.A.L. Nephrometry Score can classify a tumor's complexity-grade via the anatomical characteristics of renal masses on computerized tomography/magnetic resonance imaging. ${ }^{16,17}$ At present, there are fewer studies on the comparison of open versus laparoscopic PN through tumor's anatomical complexity grade. The purpose of this paper is to compare perioperative outcomes of laparoscopic versus open PN adopt the R.E.N.A.L. Nephrometry Score.

\section{Patients and Methods}

\section{Patients Collection}

After institutional review board consent was achieved, we identified 210 NSS procedures executed by 5 urologic oncology surgeons at the Department of Urology of the Huashan Hospital, Shanghai, China, between June 2012 and July 2015. The inclusion criteria were as follows: (1) the pathological diagnosis was renal cell carcinoma; (2) the tumor is locally solitary; (3) patients were treated with partial nephrectomy; (4) patients with complete clinical characteristics. The surgical methods included LPN (posterior abdominal approach) and OPN (through the 11th ribs). Among the 210 patients, $93(\mathrm{LPN}=52, \mathrm{OPN}=41)$ had 4-6 points (Low complexity group), 114 (LPN=67, OPN=47) had 7-9 points (Mediate complexity group), and only $3(\mathrm{OPN})$ had $10-12$ points (High complexity group), and their medical records were retrospectively reviewed. Patient demographics (gender, age), clinical characteristics (body mass index, American Society of Anesthesiologists
[ASA] score), surgical characteristics (estimated blood loss, warm ischemia time, operative time, conversion of laparoscopic procedure to open surgery), postsurgical complications (up to $30 \mathrm{~d}$ after surgery), pathologic characteristics, and postoperative length of stay were compared for each technique at different levels, respectively. Complication data were retrospectively collected through view clinical records and the evaluation of complications was audited using the modified Clavien score scale. ${ }^{18,19}$

\section{Surgical Technique}

LPN and OPN procedures were performed. All Minimally Invasive Partial Nephrectomy adopted a three- to the fourport method of a retroperitoneal laparoscopic approach. The open approach included variegated incisions between the 10th or 11th interspace, with most of them mini-flank incisions at the 11th rib. ${ }^{20}$ Vascular control was obtained with a bulldog clamp for all partial nephrectomy patients and all of them did not undergo renal artery occlusion. Reconstruction defects were accomplished with continuous suture, and parenchymal hemostasis was accomplished with absorbable hemostatic agents in bolster configuration and matrix injection. ${ }^{21}$ The complications during surgical were treated with symptomatic treatment, such as timely hemostasis when bleeding and surgical intervention for urinary leakage.

\section{Statistical Analysis}

The fundamental features of the same complexity OPN and LPN groups were computed using the Fisher exact test for categorical variables and the Mann Whitney $U$-test for continuous variables via IBM SPSS Statistics v.19. All data were presented as mean $\pm \mathrm{SD}$, and when $P$ value less than 0.05 was considered statistically significant.

\section{Results}

\section{The Demographic Characteristics of}

\section{Patients}

From June 2012 to 2015, a total of 232 cases of local single RCC who underwent PN surgery with pathological diagnosis of renal carcinoma were collected. Among these patients, 210 patients (139 males and 71 females) have complete clinical characteristics were enrolled in this study. Of these 210 patients, $93(\mathrm{LPN}=52, \mathrm{OPN}=41)$ had 4-6 points (Low complexity group), $114(\mathrm{LPN}=67, \mathrm{OPN}=47)$ had 7-9 points (Mediate complexity group), and only $3(\mathrm{OPN})$ had $10-12$ points (High complexity group). The mean age of the 
patients was 55.7 years, range from 28 to 83 years, and the mean BMI was $21.7 \mathrm{~kg} / \mathrm{m}^{2}$, range from 16.8 to $27.4 \mathrm{~kg} / \mathrm{m}^{2}$. According to the ASA score, there are 138 cases of I stage, 49 cases of II stage, and 23 cases of III stage.

In the low complexity group, 52 cases received LPN, and 41 cases received OPN. And in the mediate complexity group, 67 patients received LPN and 47 patients received OPN. Additionally, in the low complexity group, the tumor radius in patients who were treated with OPN was range from $0.50 \mathrm{~cm}$ to $2.90 \mathrm{~cm}$ (mean value: $1.39 \mathrm{~cm}$ ), and in those treated with LPN was range from $0.53 \mathrm{~cm}$ to $3.16 \mathrm{~cm}$ (mean value: $1.42 \mathrm{~cm}$ ). In the moderate complexity group, the tumor radius of OPN was range from $0.51 \mathrm{~cm}$ to $2.96 \mathrm{~cm}$ (mean value: $1.50 \mathrm{~cm}$ ) and LPN was range from $0.54 \mathrm{~cm}$ to $3.26 \mathrm{~cm}$ (mean value: $1.49 \mathrm{~cm}$ ), which has no significant statistical difference. Moreover, there is no statistical difference in the R.E.N.A.L. score between LPN and OPN in the low complexity group (4 to 6 score) and moderate complexity group (7-9 score) (Figure 1). Thus, a total of 207 patients were further analyzed.

\section{Analysis of Characteristics and Surgery Outcomes in Low Complexity Group}

Firstly, the demographic and clinical characteristics of patients who received LPN or OPN were compared. The data in Table 1 revealed that the age, gender, ASA score, BMI, TNM stage, and pathological type have no significant difference (all $P>0.05$ ).

Furthermore, perioperative characteristics in the low complexity group were analyzed. The estimated blood loss, operation time, renal artery clamp time, and postoperative length of stay showed a significant difference between OPN and LPN groups $(P<0.05$, Table 1$)$. There were no cases of intraoperative and postoperative transfusion in both LPN and
OPN groups. The results in Table 2 demonstrate that in the OPN group, estimated blood loss was about $137.1 \pm 56.1 \mathrm{~mL}$, while in the LPN group, the estimated blood loss was about $85.6 \pm 58.5 \mathrm{~mL}(P<0.001)$. The mean duration of surgery in the OPN group was about $115.1 \pm 26.6 \mathrm{~min}(55-158 \mathrm{~min})$, while in the LPN group was about $142.5 \pm 51.6 \mathrm{~min}(65-284$ $\min )(P=0.001)$. The renal artery clamp time of the OPN group was $11.9 \pm 2.6 \mathrm{~min}(7-16 \mathrm{~min})$, while in the LPN group was $17.0 \pm 4.2 \mathrm{~min}(8-25 \mathrm{~min})$. Regarding the postoperative length of stay, the average of hospitalization days was $8.0 \pm 1.9$ days in the OPN group and $7.0 \pm 1.7$ days in the LPN group $(P=0.005)$. These results revealed that patients undergoing LPN had a significantly less postoperative length of stay $(P=0.005)$ and estimated blood loss $(P<0.001)$ compared with OPN in the low complexity group.

The results in Table 1 show the postoperative complication differences of low complexity renal cortical tumors. A total of 11 cases in the OPN group had complications, 1 case of urinary leakage, and 1 case of grade III or above complications; in the LPN group, there were 7 cases with complications, 1 case of urinary leakage, and 1 case of grade III or above complications. There was no significant statistical difference between the two groups.

\section{Comparison of Characteristics and Surgery Outcomes in Mediate Complexity Group}

In the mediate complexity group, the demographic and clinical characteristics of patients who were treated with LPN or OPN have no significant difference, including age, gender, ASA score, BMI, TNM stage, and pathological type (all $P>0.05$, Table 2). As listed in Table 2, the duration of surgery time and renal artery clamp time was $154.5 \pm 48.3$ and $23.4 \pm 4.8 \mathrm{~min}$ in the LPN group,

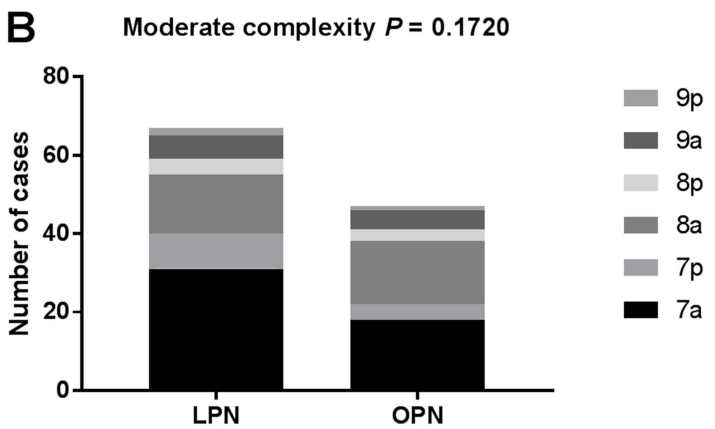

Figure I The cases of patients in LPN and OPN groups. (A) The cases of patients in different scores in LPN group. (B) The cases of patients in different scores in OPN group.

Note: Anterior (a)/posterior (p). 
Table I Perioperative and Postoperative Clinical Characteristics of Patients Treated with LPN or OPN for Low Complexity Renal Cortical Tumors

\begin{tabular}{|c|c|c|c|}
\hline \multirow[t]{2}{*}{ Parameters } & OPN & LPN & \multirow[t]{2}{*}{$P$-value } \\
\hline & $(n=4 I)(\%)$ & $(n=52)(\%)$ & \\
\hline Age (years) & $57.9 \pm 13.9$ & $57.6 \pm 9.6$ & 0.905 \\
\hline Gender (male) & $28(68.3)$ & $30(57.7)$ & 0.295 \\
\hline ASA score & & & 0.947 \\
\hline $\mathrm{I}-2$ & $37(90.2)$ & $46(88.5)$ & \\
\hline $3-4$ & $4(9.8)$ & $6(11.5)$ & \\
\hline BMI $\left(\mathrm{kg} / \mathrm{m}^{2}\right)$ & $22.5 \pm 2.9$ & $21.5 \pm 2.7$ & 0.867 \\
\hline TNM stage & & & 0.448 \\
\hline pTla & 38 & 51 & \\
\hline pTIb & 3 & $\mathrm{I}$ & \\
\hline Pathological type & & & 0.200 \\
\hline Clear cell & 35 & 49 & \\
\hline Papillary & 4 & 3 & \\
\hline Chromophobe & 2 & 0 & \\
\hline Estimated blood loss (mL) & $|37.| \pm 56 . \mid$ & $85.6 \pm 58.5$ & $<0.001$ \\
\hline Duration of surgery (min) & $|I 5| \pm 26.6$. & $|42.5 \pm 5| .6$ & 0.001 \\
\hline Transfusion & 0 & 0 & - \\
\hline Renal artery clamp time (min) & $11.9 \pm 2.6$ & $17.0 \pm 4.2$ & $<0.001$ \\
\hline Conversion to open & - & 2 & - \\
\hline Postoperative length of stay (d) & $8.0 \pm 1.9$ & $7.0 \pm 1.7$ & 0.005 \\
\hline Positive surgical margin & $\mathrm{I}(2.4)$ & $2(3.8)$ & 0.703 \\
\hline Any complication & II (26.8) & $7(13.5)$ & 0.105 \\
\hline Grade $\geq 3$ complications & $\mathrm{I}(2.4)$ & $\mathrm{I}(\mathrm{I} .9)$ & 0.865 \\
\hline Any urine leak & $\mathrm{I}(2.4)$ & $\mathrm{I}(\mathrm{I} .9)$ & 0.865 \\
\hline Urine leak requiring intervention & 0 & 0 & - \\
\hline
\end{tabular}

Abbreviations: OPN, open partial nephrectomy; LPN, Laparoscopic partial nephrectomy; ASA, American Society of Anesthesiologists; BMI, body mass index.

respectively, while the data were $121.0 \pm 26.0$ and $16.2 \pm$ $5.3 \mathrm{~min}$ in the OPN group. These results revealed that LPN had notably longer renal artery clamp time $(P<0.001)$ and operative time $(P<0.001)$ compared with OPN.

There were no statistically significant differences in the rates of complications, estimated blood loss, and postoperative length of stay between LPN and OPN (all $P>0.05$, Table 2).

\section{Discussion}

The anatomy of RCC, such as diameter, depth of growth, and hilar vessel location, is considered an important predictor of surgical complexity and the occurrence of postoperative complications in partial nephrectomy for RCC. $^{22,23}$ In 2009, Alexander Kutikov and co-workers proposed the R.E.N.A.L scoring system based on RCC radius $(\mathrm{R})$, exophytic/endophytic properties of the tumor
(E), nearness of tumor deepest portion to the collecting system or sinus (N), anterior (a)/posterior (p) descriptor (A) and the location relative to the polar line (L). ${ }^{16}$ The R. E.N.A.L scoring system can not only accurately classify RCC into low complexity group, mediate complexity group, and high complexity group, but also predict intraoperative, postoperative outcomes and complications for patients with partial nephrectomy. ${ }^{24,25}$ At present, few studies are comparing laparoscopic and open partial nephrectomy for RCC based on R.E.N.A.L score, and there is no direct objective basis for whether LPN or OPN should be chosen for patients to undergo partial nephrectomy surgery.

A previous study reported that patients treated with LPN exhibited shorter surgical time, less blood loss, shorter hospital stay, faster postoperative recovery time, but longer intraoperative warm ischemia time compared 
Table 2 Perioperative and Postoperative Clinical Characteristics of Patients Treated with Partial Nephrectomy for Moderate Complexity Renal Cortical Tumors

\begin{tabular}{|c|c|c|c|}
\hline \multirow[t]{2}{*}{ Parameters } & OPN & LPN & \multirow[t]{2}{*}{$P$-value } \\
\hline & $(n=47)(\%)$ & $(n=67)(\%)$ & \\
\hline Age (years) & $54.4 \pm 13.7$ & $53.8 \pm 12.6$ & 0.821 \\
\hline Gender (male) & $33(70.2)$ & $53(79.1)$ & 0.278 \\
\hline $\begin{array}{l}\text { ASA score } \\
\qquad \begin{array}{l}1-2 \\
3-4\end{array}\end{array}$ & $\begin{array}{c}43(91.5) \\
4(8.5)\end{array}$ & $\begin{array}{c}61(91.0) \\
6(9.0)\end{array}$ & 0.934 \\
\hline BMI $\left(\mathrm{kg} / \mathrm{m}^{2}\right)$ & $21.2 \pm 2.5$ & $21.9 \pm 2.7$ & 0.223 \\
\hline $\begin{array}{l}\text { TNM stage } \\
\text { PTIa } \\
\text { PTIb }\end{array}$ & $\begin{array}{c}41 \\
6\end{array}$ & $\begin{array}{c}64 \\
3\end{array}$ & 0.207 \\
\hline $\begin{array}{l}\text { Pathological type } \\
\text { Clear cell } \\
\text { Papillary } \\
\text { Chromophobe }\end{array}$ & $\begin{array}{l}41 \\
4 \\
2\end{array}$ & $\begin{array}{c}60 \\
6 \\
1\end{array}$ & 0.662 \\
\hline Estimated blood loss (mL) & $181.2 \pm 103.4$ & $165.5 \pm 76.5$ & 0.356 \\
\hline Duration of surgery (min) & $121.0 \pm 26.0$ & $154.5 \pm 48.3$ & $<0.001$ \\
\hline Transfusion & $8(17.0)$ & $4(6)$ & 0.114 \\
\hline Renal artery clamp time $(\mathrm{min})$ & $16.2 \pm 5.3$ & $23.4 \pm 4.8$ & $<0.001$ \\
\hline Conversion to open & - & 5 & - \\
\hline Postoperative length of stay (d) & $8.2 \pm 1.9$ & $8.3 \pm 2.3$ & 0.118 \\
\hline Positive surgical margin & I (2.I) & $3(4.5)$ & 0.529 \\
\hline Any complication & $14(29.8)$ & $20(29.8)$ & 0.994 \\
\hline Grade $\geq 3$ complications & $2(14.2)$ & $4(6.0)$ & 0.686 \\
\hline Any urine leak & $2(4.3)$ & $4(6.0)$ & 0.686 \\
\hline Urine leak requiring intervention & $\mathrm{I}(2.1)$ & $2(3.0)$ & 0.778 \\
\hline
\end{tabular}

Abbreviations: OPN, open partial nephrectomy; LPN, Laparoscopic partial nephrectomy; ASA, American Society of Anesthesiologists; BMI, body mass index.

with those treated with OPN in 200 consecutive patients with a sporadic single renal tumor. ${ }^{26}$ The study by Gholam Hossein Rezaeetalab and coworkers compared the difference between OPN and LPN in a group of patients over 18 years old with a single renal mass of less than $4 \mathrm{~cm}$, the results indicated that LPN has some benefits over OPN, including decreased postoperative pain and higher patient satisfaction. $^{27}$ In the present study, the patients were divided into low complex renal tumor group, mediate complex group, and highly complex group according to the R.E.N.A.L score. All patients in the highly complex group were treated with OPN. Thus, further analyses were carried out in low and mediate complex groups, respectively. The results showed that LPN had less bleeding than the OPN group in both the low complexity group and mediate complexity group, which were generally consistent with the results in a previous study. ${ }^{26}$ These data reflected the characteristics of LPN with fine anatomy, clear vision, and less damage to the tissues and blood vessels surrounding the lesion.

The current study also observed that in terms of duration of surgery and renal artery clamp time, LPN exhibited longer time than OPN in both the low complexity group and mediate complexity group, which mainly because LPN renal reconstruction is more difficult than OPN with less space for operation and more time required for changing suture instruments, and also related to the operator's proficiency. Another single-center study also observed that the LPN group had higher warm ischemia time, intraoperative estimated blood loss, and operation duration, but exhibited shorter hospitalization time and less severe complications than the OPN group. $^{28}$ Whereas, both the 
operative time and warm ischemia time are within an acceptable time frame, and many studies demonstrated that there is no significant difference in long-term glomerular filtration rate, ${ }^{29,30}$ and that operative time and warm ischemia time are not constraining factors in the choice of LPN.

In the present study, the results also exhibited that the mean postoperative hospital stay of LPN was significantly less than that of OPN in the R.E.N.A.L score low complex group. In the moderate complex R.E.N.A.L group, the mean hospital stays time after treatment of LPN was slightly longer than that of OPN, but there was no statistically significant difference. LPN has the advantage of quick postoperative recovery in RCCs with low R.E.N.A. L scores, but not in RCC patients with moderate R.E.N.A. $\mathrm{L}$ scores. The postoperative related complications in LPN and OPN groups in both low and moderate complex groups have no significant difference. These results were in accordance with previous studies about the results between OPN and LPN treated groups. ${ }^{29,31}$ A recent study by Salah $\mathrm{M}$ and co-workers indicated that a newly modified nephelometry score (MNS) was associated with better prediction of the outcome of partial nephrectomy when compared to R.E.N.A.L. ${ }^{32}$ In future studies, the data will try to be further compared by using the newly modified nephelometry score.

This study is a non-clinical randomized controlled retrospective case study and there are some limitations. For instance, there may be some bias in the statistics in terms of clinical data and measurement of imaging data. There is no accurate and complete data of preoperative and postoperative renal function, as well as complete data of glomerular filtration rate. Recent studies reported that off-clamp and onclamp robotic partial nephrectomy (RPN) are safe approaches and have negligible impact on renal function. ${ }^{33,34}$ Furthermore, robot-assisted laparoscopic partial nephrectomy (RALPN) has been carried out in our hospital. In the future, RALPN, LPN, and OPN can be compared, and patient's glomerular filtration rate, renal function, quality of life, and survival rate can be followed up for follow-up related studies.

\section{Conclusion}

In conclusion, LPN has obvious advantages in intraoperative bleeding and postoperative recovery. There is no significant difference between LPN and OPN in postoperative related complications. RCC patients with R.E. N.A.L. score (4-6) are recommended to be treated with
LPN as the first choice. R.E.N.A.L. score for moderate complex renal tumors, LPN has no significant difference in postoperative complications and postoperative recovery compared with OPN and has a longer operative time and warm ischemia time. Thus, for patients with moderate complex renal tumors, the surgeon can choose the surgical approach based on his own experience and expertise. The present study provides a basis for the clinical treatment of RCC.

\section{Ethics Statement}

All procedures performed in studies involving human participants were in accordance with the ethical standards of the Shanghai Huashan Hospital research committee and with the 1964 Helsinki declaration and its later amendments or comparable ethical standards. Each participant signed written informed consent.

\section{Disclosure}

The authors report no conflicts of interest in this work.

\section{References}

1. Arabsalmani M, Mohammadian-Hafshejani A, Ghoncheh M, et al. Incidence and mortality of kidney cancers, and human development index in Asia; a matter of concern. J Nephropathol. 2016;6(1):30-42. doi:10.15171/jnp.2017.06

2. Ljungberg B, Bensalah $\mathrm{K}$, Canfield $\mathrm{S}$, et al. EAU guidelines on renal cell carcinoma: 2014 update. Eur Urol. 2015;67(5):913-924. doi:10.1016/j.eururo.2015.01.005

3. Zhang Y, Ellinger J. Clinical studies applying cytokine-induced killer cells for the treatment of renal cell carcinoma. Cancers. 2020;12 (9):2471.

4. Simone G, Tuderti G, Anceschi U, et al. Oncological outcomes of minimally invasive partial versus minimally invasive radical nephrectomy for cT1-2/N0/M0 clear cell renal cell carcinoma: a propensity score-matched analysis. World $J$ Urol. 2017;35(5):789-794. doi:10.1007/s00345-016-1923-2

5. Koo KC, Kim JC, Cho KS, et al. Oncological outcomes after partial vs radical nephrectomy in renal cell carcinomas of $<=7 \mathrm{~cm}$ with presumed renal sinus fat invasion on preoperative imaging. BJU Int. 2016;117(1):87-93.

6. Suo X, Chen J, Zhao Y, et al. Clinicopathological and radiological significance of the collateral vessels of renal cell carcinoma on preoperative computed tomography. Sci Rep. 2021;11(1):5187. doi:10.1038/s41598-021-84631-w

7. Ebbing J, Menzel F, Frumento P, et al. Outcome of kidney function after ischaemic and zero-ischaemic laparoscopic and open nephron-sparing surgery for renal cell cancer. BMC Nephrol. 2019;20 (1):40. doi:10.1186/s12882-019-1215-3

8. Mina-Riascos SH, Vitagliano G. Effectiveness and safety of partial nephrectomy-no ischemia vs. warm ischemia: systematic review and meta-analysis. Invest Clin Urol. 2020;61(5):464-474. doi:10.4111/ icu. 20190313

9. Liang W, Li J, Zhang W, et al. Prolonged postoperative ileus in gastric surgery: is there any difference between laparoscopic and open surgery? Cancer Med. 2019;8(12):5515-5523. doi:10.1002/cam4.2459 
10. Sawinski D, Locke JE. Evaluation of kidney donors: core curriculum 2018. Am J Kidney Dis. 2018;71(5):737-747. doi:10.1053/j. ajkd.2017.10.018

11. Marszalek M, Meixl H, Polajnar M, Rauchenwald M, Jeschke K, Madersbacher S. Laparoscopic and open partial nephrectomy: a matched-pair comparison of 200 patients. Eur Urol. 2009;55 (5):1171-1178. doi:10.1016/j.eururo.2009.01.042

12. Zhou S, Wang X, Zhao C, et al. Laparoscopic vs open colorectal cancer surgery in elderly patients: short- and long-term outcomes and predictors for overall and disease-free survival. BMC Surg. 2019;19 (1):137. doi:10.1186/s12893-019-0596-3

13. Kim JJ, Lim JY, Nguyen SQ. Laparoscopic resection of gastrointestinal stromal tumors: does laparoscopic surgery provide an adequate oncologic resection? World J Gastrointest Endosc. 2017;9 (9):448-455. doi:10.4253/wjge.v9.19.448

14. Kamel AI, Badawy MH, Elganzoury H, et al. Clinical versus pathologic staging of renal tumors: role of multi-detector CT urography. Electron Physician. 2016;8(1):1791-1795. doi:10.19082/1791

15. Manno S, Dell'Atti L. Safety and efficacy of transperitoneal laparoscopic nephron sparing surgery in patients with previous abdominal surgery. Urologia J. 2021;88(1):14-20.

16. Kutikov A, Uzzo RG. The R.E.N.A.L. Nephrometry Score: a comprehensive standardized system for quantitating renal tumor size, location and depth. J Urol. 2009;182(3):844-853. doi:10.1016/j. juro.2009.05.035

17. Chen SH, Wu YP, Li XD, et al. R.E.N.A.L. Nephrometry Score: a preoperative risk factor predicting the Fuhrman grade of clear-cell renal carcinoma. $J$ Cancer. 2017;8(18):3725-3732. doi:10.7150/ jca. 21189

18. Sprenkle PC, Power N, Ghoneim T, et al. Comparison of open and minimally invasive partial nephrectomy for renal tumors 4-7 centimeters. Eur Urol. 2012;61(3):593-599. doi:10.1016/j. eururo.2011.11.040

19. Wang H, Zhou L, Guo J, et al. Mini-flank supra-12th rib incision for open partial nephrectomy compared with laparoscopic partial nephrectomy and traditional open partial nephrectomy. PLoS One. 2014;9(2):e89155. doi:10.1371/journal.pone.0089155

20. Russo P, Mano R. Open mini-flank partial nephrectomy: an essential contemporary operation. Korean J Urol. 2014;55(9):557-567. doi:10.4111/kju.2014.55.9.557

21. Nogueira L, Katz D, Pinochet R, Kurta JM, Coleman JA. Comparison of gelatine matrix-thrombin sealants used during laparoscopic partial nephrectomy. BJU Int. 2008;102(11):1670-1674. doi:10.1111/j.1464-410X.2008.07926.X

22. Han Y, Shao N, Xi X, Hao X. Use of microwave ablation in the treatment of patients with multiple primary malignant tumors. Thorac Cancer. 2017;8(4):365-371. doi:10.1111/1759-7714.12445
23. Zhao PT, Richstone L, Kavoussi LR. Laparoscopic partial nephrectomy. Int J Surg. 2016;36(Pt C):548-553. doi:10.1016/j. ijsu.2016.04.028

24. Acosta Ruiz V, Ladjevardi S, Brekkan E, et al. Periprocedural outcome after laparoscopic partial nephrectomy versus radiofrequency ablation for T1 renal tumors: a modified R.E.N.A.L Nephrometry Score adjusted comparison. Acta Radiol. 2019;60(2):260-268. doi:10.1177/0284185118780891

25. Zhou HJ, Yan Y, Zhang JZ, Liang LR, Guo SB. Role of R.E.N.A.L. Nephrometry Score in laparoscopic partial nephrectomy. Chin Med $J$ (Engl). 2017;130(18):2170-2175. doi:10.4103/0366-6999.213973

26. Gill IS, Matin SF, Desai MM, et al. Comparative analysis of laparoscopic versus open partial nephrectomy for renal tumors in 200 patients. $J$ Urol. 2003;170(1):64-68. doi:10.1097/01. ju.0000072272.02322.ff

27. Rezaeetalab GH, Karami H, Dadkhah F, Simforoosh N, Shakhssalim N. Laparoscopic versus open partial nephrectomy for stage T1a of renal tumors. Urol J. 2016;13(6):2903-2907.

28. Kartal I, Karakoyunlu N, Çakici M, Karabacak O, Sağnak L, Ersoy H. Oncological and functional outcomes of open versus laparoscopic partial nephrectomy in T1b tumors: a single-center analysis. Int Braz J Urol. 2020;46(3):341-350. doi:10.1590/s1677-5538. ibju.2018.0865

29. You C, Du Y, Wang H, et al. Laparoscopic versus open partial nephrectomy: a systemic review and meta-analysis of surgical, oncological, and functional outcomes. Front Oncol. 2020;10:583979. doi: $10.3389 /$ fonc. 2020.583979

30. Springer C, Hoda MR, Fajkovic H, et al. Laparoscopic vs open partial nephrectomy for T1 renal tumours: evaluation of long-term oncological and functional outcomes in 340 patients. BJU Int. 2013;111(2):281-288. doi:10.1111/j.1464-410X.2012.11280.X

31. Lucas SM, Mellon MJ, Erntsberger L, Sundaram CP. A comparison of robotic, laparoscopic and open partial nephrectomy. JSLS. 2012;16 (4):581-587.

32. Salah M, ElSheemy MS, Ghoneima W, et al. Modified R.E.N.A.L nephrometry score for predicting the outcome following partial nephrectomy. Afr J Urol. 2020;26(1):45. doi:10.1186/s12301-02000056-3

33. Bertolo R, Simone G, Garisto J, et al. Off-clamp vs on-clamp robotic partial nephrectomy: perioperative, functional and oncological outcomes from a propensity-score matching between two high-volume centers. Eur J Surg Oncol. 2019;45(7):1232-1237. doi:10.1016/j. ejso.2018.12.005

34. Simone G, Misuraca L, Tuderti G, et al. Purely off-clamp robotic partial nephrectomy: preliminary 3-year oncological and functional outcomes. Int J Urol. 2018;25(6):606-614. doi:10.1111/iju.13580
Cancer Management and Research

\section{Publish your work in this journal}

Cancer Management and Research is an international, peer-reviewed open access journal focusing on cancer research and the optimal use of preventative and integrated treatment interventions to achieve improved outcomes, enhanced survival and quality of life for the cancer patient.
The manuscript management system is completely online and includes a very quick and fair peer-review system, which is all easy to use. Visit http://www.dovepress.com/testimonials.php to read real quotes from published authors. 